Methods Based on the international and domestic guidelines for media reports, we developed a checklist to assess adherence of Chinese media reports to recommended principles. We adopted a seed approach to search influential violence stories. Reports were assessed using the unified coding scheme by different coders; the conflicts between raters were resolved through discussion.

Results In total, 17,222 media reports were captured; 788 met inclusion criteria. $49.5 \%$ and $52.7 \%$ of 788 included reports mentioned real and complete names of writer and editor, respectively. of 329 reports including quotes or opinions from doctors, patients, and hospital administrative personnel, only $15.79 \%, 11.25 \%$ and $18.62 \%$ respectively specified they had obtained the consent of the interviewees. $21.6 \%$ of reports including all specifically-coded details of the violent acts against doctors (time, place, methods and perpetrators), and $43.5 \%$ of reports included some details. Only $18.7 \%$ of media reports offered suggestions for seeking professional help in case doctors are threatened by violence and $11.3 \%$ offered contact information for third parties who mediate doctorpatient conflicts.

Conclusion Most internet-based media reports did not strictly follow media recommendations from authoritative bodies. Strong efforts are needed to require journalists to increase adherence so risk of violence against doctors is reduced in China.

Learning Outcomes Most Chinese internet-based media sources did not appropriately report stories concerning violence against doctors.

\section{P2.004 A SCOPING REVIEW ON STRATEGIES AND INTERVENTIONS IN GLOBAL NEUROTRAUMA PREVENTION}

\begin{abstract}
1,2Santhani M Selveindran*, 1,2Peter Hutchinson, ${ }^{3}$ Tamara Tango, ${ }^{2,4}$ Muhammad Mukhtar Khan, ${ }^{2,4}$ Daniel Simadibrata, ${ }^{2,5}$ Carol Brayne, ${ }^{2,5}$ Christine Hill, ${ }^{1,2}$ Angelos Kolias, 2,6 Franco Servadei, ${ }^{1,2}$ Alexis Joannides, ${ }^{2,7}$ Andres Rubiano, ${ }^{2,8} \mathrm{Hamisi}$ Shabani. ${ }^{1}$ Cambridge University Hospitals Trust, Cambridge, UK; ${ }^{2}$ NIHR Global Health Research Group on Neurotrauma, Cambridge, UK; ${ }^{3}$ Universitas Indonesia, Depok, Indonesia; ${ }^{4}$ Northwest School of Medicine and Gernal Hospital and Research Centre, Peshawar, Pakistan; ${ }^{5}$ Cambridge Institute of Public Health, Cambridge, UK; ${ }^{6}$ Humanitas University and Research Hospital, Milan, Italy; ${ }^{7}$ Neuroscience Institute El Bosque University, Bogota, Colombia; ${ }^{8}$ Muhimbili Orthopaedic Institute and Muhimbili University College of Allied Health Sciences, Dar es Salaam, Tanzania
\end{abstract}

\subsection{6/injuryprev-2021-safety.94}

Background Neurotrauma from road traffic collisions (RTCs) is an important yet preventable cause of death and disability especially in low and middle income countries (LMICs). However, it is uncertain if current preventative strategies are applicable globally due to variations in environment, resources, population, culture and infrastructure. This scoping review aims to identify, quantify and describe the evidence on approaches in neurotrauma and RTCs prevention, and ascertain contextual factors that influence their implementation in LMICs and high income countries (HICs).

Methods A systematic search was conducted using five electronic databases, grey literature databases, and bibliographic and citation searching of selected articles. The extracted data were presented using figures, tables, and accompanying narrative summaries.

Results A total of 411 publications met the inclusion criteria. Only 65 papers came from LMICs, which mostly described primary prevention, focussing on road safety. For the reviews, 41 papers $(66.1 \%)$ reviewed primary, 18 tertiary $(29.1 \%)$, and three secondary preventative approaches. Most of the primary papers in the reviews came from HICs (67.7\%) with 5 reviews on only LMIC papers. Fifteen reviews (24.1\%) included papers from both HICs and LMICs. Contextual factors were described in 62 papers, and varied depending on the interventions.

Conclusions There is a large quantity of global evidence on strategies and interventions for neurotrauma and RTCs prevention. However, less papers were from LMICs, especially on secondary and tertiary prevention. More primary research needs to be done in these countries to determine what strategies and interventions exist, and the applicability of HIC interventions in LMICs.

\section{P2.005 EPIDEMIOLOGY OF MAJOR TRAUMA IN NEW ZEALAND: A LITERATURE REVIEW}

'Luisa Montoya*, 'Bridget Kool, ${ }^{2}$ Gabrielle Davie, ${ }^{3}$ Bridget Dicker. ' University of Auckland, Auckland, New Zealand; ' University of Otago, Dunedin, New Zealand; ${ }^{3}$ St John, Auckland, New Zealand

\subsection{6/injuryprev-2021-safety.95}

Background Traumatic injuries are one of the major causes of disability and death worldwide and have an immense impact on the health of the population. In New Zealand, an estimated $8 \%$ of total health loss from all causes was attributed to injuries in 2016. The aim of this research is to describe the incidence and characteristics of major trauma in New Zealand up to May 2019.

Methods A literature review was performed in MEDLINE, EMBASE, CINAHL and Scopus. Studies published in English reporting the incidence of major trauma in New Zealand were included. Major trauma was defined as death or an Injury Severity Score greater than 12 . The quality of studies was assessed using the GATE LITETM tool.

Results Thirty-one studies fulfilled the inclusion criteria. The majority of studies were descriptive observational studies $(n=29)$. The population incidence rate of fatal major trauma was highest among those injured from motor vehicle crashes or falls, among Māori males and those sustaining head injuries. The incidence rate of non-fatal major trauma was highest among young Māori males. Length of hospital stay was greatest in patients with the highest Injury Severity Scores.

Conclusions The incidence of major trauma varies by age, sex and ethnicity. The review findings highlight the need for further analytical studies that can explore factors that may impact on survival from major trauma.

Learning Outcomes Capacity to integrate knowledge and to describe the different aspects of major trauma.

\section{P2.006 KNOWLEDGE AND ATTITUDE IN TRAUMA CARE: A SURVEY ON MOZAMBIQUE'S CENTRAL HOSPITALS}

Jessica Matos*. Eduardo Mondlane University, Maputo, Mozambique

\subsection{6/injuryprev-2021-safety.96}

Severe or major trauma is a pandemic and one of the leading causes of death and disability; It is distributed unequally worldwide with most of these deaths occurring in LMICs. 